\title{
Annular rectal constriction caused by infiltrating bladder cancer: A case report
}

\author{
HISASHI TAKEUCHI, NAOTO TOKUYAMA, ISAO KURODA and TEIICHIRO AOYAGI \\ Department of Urology, Tokyo Medical University Ibaraki Medical Center, Ibaraki 300-0395, Japan
}

Received August 16, 2016; Accepted September 15, 2016

DOI: $10.3892 / \operatorname{mco} .2016 .1047$

\begin{abstract}
A 69-year-old man was referred to our hospital with chief complaints of urinary incontinence, pollakiuria, thin stools and edema of the left lower extremity. Computed tomography and magnetic resonance imaging revealed bilateral hydronephrosis, thickening of the rectal and posterolateral bladder walls, and enlargement of the left obturator lymph nodes, suggesting metastasis. The carcinoembryonic antigen and carbohydrate antigen $19-9$ levels were $5.6 \mathrm{ng} / \mathrm{ml}$ and $460.1 \mathrm{U} / \mathrm{ml}$, respectively. A transurethral bladder biopsy revealed grade 2 urothelial carcinoma with glandular differentiation. A transrectal needle biopsy suggested cancer infiltration of the rectal wall and an annular rectal constriction caused by an infiltrating bladder cancer was diagnosed. Three courses of gemcitabine and cisplatin chemotherapy were administered and demonstrated good efficacy.
\end{abstract}

\section{Introduction}

Invasive bladder cancers usually metastasize to the lymph nodes, liver, lung and bone. Furthermore, aggressive local invasion into adjacent structures is also commonly observed (1). However, the occurrence of rectal stricture due to infiltration by bladder cancer is relatively rare. We herein report the case of a patient who presented with an aggressive bladder cancer that resulted in rectal infiltration and partial obstruction.

\section{Case report}

A 69-year-old man was referred to the Tokyo Medical University Ibaraki Medical Center (Inashiki, Japan) with chief complaints of urinary incontinence and pollakiuria. The patient also had thin stools and edema of the left lower extremity. The patient had a history of hypertension and a

Correspondence to: Dr Hisashi Takeuchi, Department of Urology, Tokyo Medical University Ibaraki Medical Center, 3-20-1 Chuo, Ami-machi, Inashiki-gun, Ibaraki 300-0395, Japan

E-mail: t-hisa@tokyo-med.ac.jp

Key words: bladder cancer, rectal obstruction, chemotherapy family history of prostate and gastric cancer occurring in his father and daughter, respectively.

Computed tomography (CT) and magnetic resonance imaging (MRI) revealed bilateral hydronephrosis and thickening of the wall of the rectum and the posterolateral wall of the bladder. Enlargement of the left obturator lymph nodes was also observed, suggesting the presence of lymph node metastasis (Figs. 1 and 2).

The laboratory test results were as follows: Normal blood count; creatinine level, $1.16 \mathrm{mg} / \mathrm{dl}$; estimated glomerular filtration rate, $48.9 \mathrm{ml} / \mathrm{min} / 1.73 \mathrm{~m}^{2}$; carcinoembryonic antigen (CEA) level, $5.6 \mathrm{ng} / \mathrm{ml}$ (normal range, $<5.0 \mathrm{ng} / \mathrm{ml}$ ); and carbohydrate antigen (CA) 19-9 level, $460.1 \mathrm{U} / \mathrm{ml}$ (normal range, $<37.0 \mathrm{U} / \mathrm{ml}$ ). Urinalysis revealed the presence of 1-4 white and 0-1 red blood cells per high-power field in the urine. Furthermore, the urine cytology was class IIIb. Cystoscopic examination revealed small papillary tumors and a pale thickened bladder mucosa at the retrotrigonum of the bladder, involving the ureteral orifices bilaterally. These results suggested extrinsic invasion of the bladder by the tumor. Rectal examination indicated a narrowed rectal lumen, with an intact rectal mucosa. Colonoscopy revealed a narrow rectal lumen with an edematous mucosa, suggesting extrinsic compression. Transurethral biopsy of the papillary lesions and the thickened bladder wall revealed the presence of grade 2 urothelial carcinoma with glandular differentiation (Fig. 3). Transrectal bladder needle biopsy suggested that the bladder cancer cells had infiltrated the rectal wall.

Three courses of gemcitabine and cisplatin (GC) chemotherapy were administered and this therapy continued to demonstrate effectiveness post-treatment. A CT scan revealed an improvement in the thickened bladder and rectal walls (Fig. 4). The CEA and CA19-9 levels were also reduced to $2.3 \mathrm{ng} / \mathrm{ml}$ and $66.1 \mathrm{U} / \mathrm{ml}$, respectively. The symptom of thin stools also subsided. At the last follow-up 6 months after chemotherapy, the disease status was stable.

Informed consent was obtained form the patient regarding the publication of the case details.

\section{Discussion}

Prostate cancer is reported to be the most common cause of rectal obstruction among urogenital cancers (2). However, annular constriction of the rectum secondary to bladder cancer has rarely been reported. There are three main characteristics 

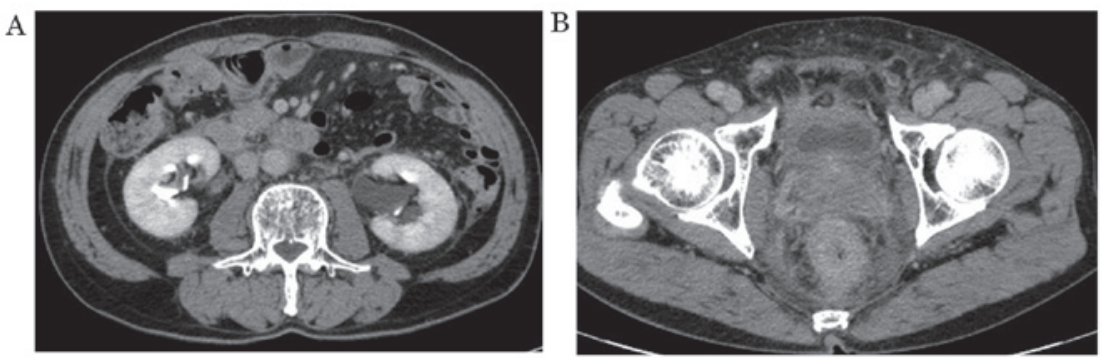

Figure 1. Abdominal computed tomography showing (A) bilateral hydronephrosis and (B) thickening of the posterolateral bladder and rectal walls.
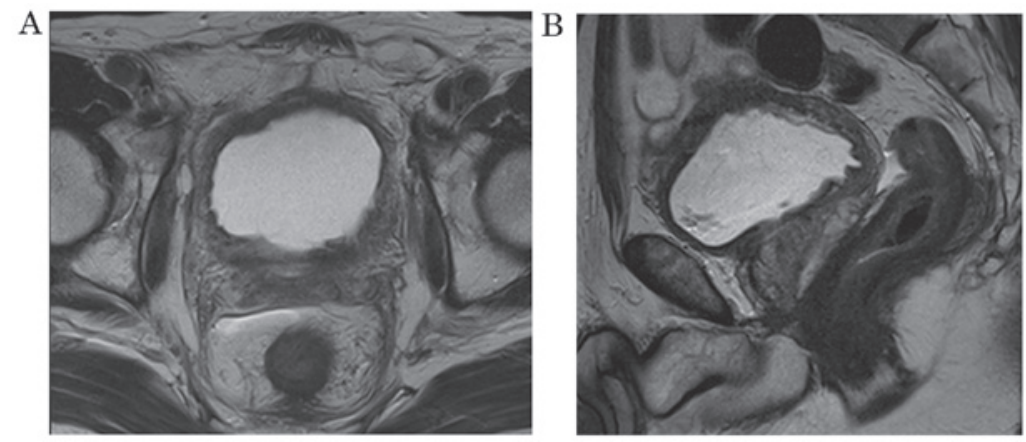

Figure 2. Pelvic magnetic resonance imaging [(A) axial; (B) sagittal] showing bilateral hydronephrosis, thickening of the posterolateral bladder and rectal wall, and enlargement of the left obturator lymph nodes.
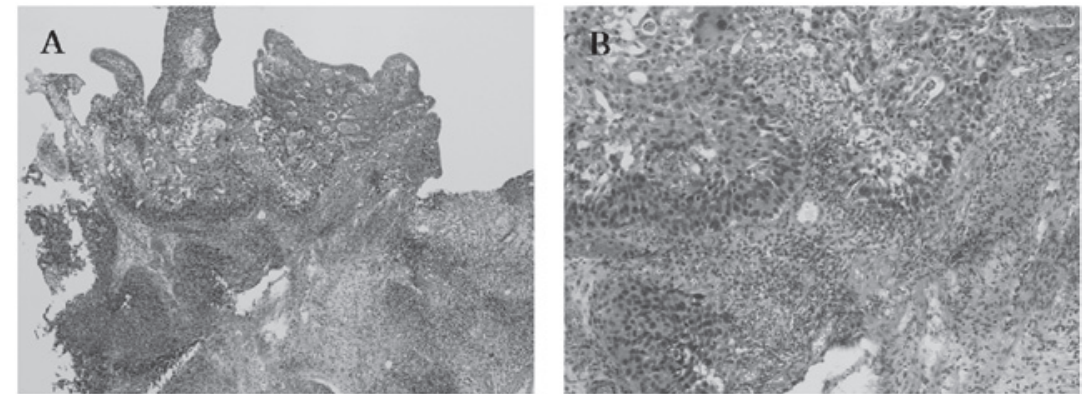

Figure 3. Histopathological examination revealed grade 2 urothelial carcinoma with glandular differentiation [hematoxylin and eosin stain, original magnification, (A) $\mathrm{x} 4$ and (B) $\mathrm{x} 20]$.

of an annular rectal constriction caused by an infiltrating bladder cancer: The majority of the patients are male, the tumor is of high grade and stage, and the prognosis is poor (3-6). The characteristics of the patient in the present case, who was diagnosed with grade 2 stage IV urothelial carcinoma, were consistent with the previously reported cases.

The mechanism underlying annular rectal stricture caused by bladder cancer remains unknown. Stillwell et al (3) reported two cases of male patients with annular constrictions of the rectum and they hypothesized that a locally aggressive cancer of the bladder neck or trigonum may break through the Denonvilliers' fascia and encircle the rectum. Langenstroer et al (4) described another case with similar rectal obstruction that developed 3 years after cystoprostatectomy; they suggested that surgical deposition of cancer cells may be the cause of the rectal obstruction.

Kobayashil et al (5) reported three cases of male patients exhibiting annular constriction of the rectum due to the

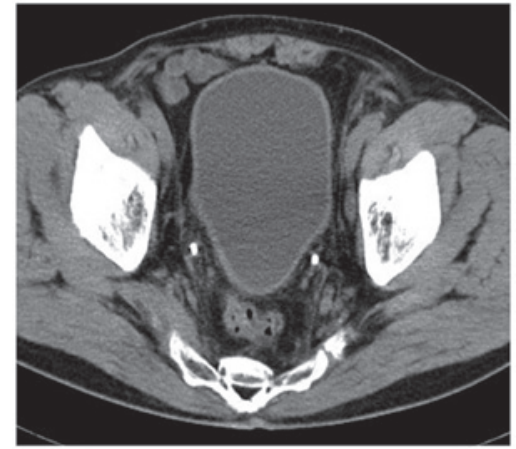

Figure 4. Abdominal computed tomography scan showing an improvement of the thickened bladder and rectal walls.

infiltration by a bladder cancer. All these cases exhibited progressive spread of tumor cells involving the bladder wall. The infiltrating tumor, encroaching both ureteral orifices, 
resulted in reduced bladder capacity. The $\mathrm{CT}$ results showing thickened lateral pedicles without involvement of the perirectal fat layer were intriguing. Therefore, Kobayashil et al (5) hypothesized that cancer cells of this type were able to easily spread along the lateral pedicles to reach the posterior rectal wall and then infiltrate the rectal wall.

In the present case, the CT findings of the enlargement of the left obturator lymph nodes, without the involvement of the perirectal fat layer, were of interest. These results differed from those of the surgical deposition of cancer cells that caused rectal constriction. Therefore, we hypothesized that, consistent with the report by Kobayashil et al (5), the cancer cells in this study may have spread along the lateral pedicles to reach the posterior rectal wall prior to penetrating it.

Almost all reported cases of annular rectal constrictions caused by an infiltrating bladder cancer are associated with a poor prognosis. By contrast, our patient responded well to $\mathrm{GC}$ chemotherapy. Therefore, immediate initiation of an appropriate chemotherapy following diagnosis may be beneficial and achieve a good response, given that a complete response to treatment may not be feasible.

In conclusion, annular rectal constriction is a rare complication of bladder cancer and is associated with a poor prognosis. It is important that physicians consider the possibility of an annular rectal constriction caused by an infiltrating bladder cancer when patients with bladder cancer complain of thin stools and the clinical examination reveals narrowing of the rectal lumen. Immediate chemotherapy initiation should be considered in such cases.

\section{Acknowledgements}

The authors would like to thank Enago (www.enago.jp) for reviewing the English language of the original manuscript.

\section{References}

1. Tabbara WS and Mehio AR: Metastatic patterns of bladder carcinoma. Prog Clin Biol Res 162A: 145-160, 1984.

2. Fry DE, Amin M and Harbrecht PJ: Rectal obstruction secondary to carcinoma of the prostate. Ann Surg 189: 488-492, 1979.

3. Stillwell TJ, Rife CC and Lieber MM: Bladder carcinoma presenting with rectal obstruction. Urology 34: 238-240, 1989.

4. Langenstroer P, Zacharias A, Almagro U and Dewire D: Annular constriction of the rectum secondary to transitional cell carcinoma of the bladder. Urology 47: 442-444, 1996.

5. Kobayashi S, Kato H, Iijima K, Kinebuchi Y, Igawa Y and Nishizawa O: Annular rectal constriction due to infiltration by bladder cancer. Hinyokika Kiyo 52: 569-572, 2006.

6. Abol-Enein H, El-Baz M, Abd El-Hameed MA, Abdel-Latif M and Ghoneim MA: Lymph node involvement in patients with bladder cancer treated with radical cystectomy: A patho-anatomical study-a single center experience. J Urol 172: 1818-1821, 2004. 